\section{P32 (continued)}

and seek assistance when needed. Students demonstrated lowest confidence in the ability to review medications, identify drug/nutrient interactions, and demonstrate negotiation skills in the clinical setting.

Conclusion: First year students demonstrated lower selfefficacy, even at the graduate level; however, self-efficacy was higher in students after completing supervised practice. This work identifies potential areas of growth in dietetics programs and highlights the need for experiences that enhance self-efficacy.

Funding: None.

\section{P33 Sensory Perceptions of Cancer Survivors and Their Caregivers of Garden-Harvested and Grocery-Purchased Produce}

Ashlea Braun, $P h D, L D, R D N$, Medical Dietetics \& Health Sciences, School of Health and Rehabilitation Sciences, The Ohio State University College of Medicine; Christopher Simons, PhD, Department of Food Science \& Technology, The Ohio State University; Jessica Kilbarger, MS, LD, RDN, Medical Dietetics \& Health Sciences, School of Health and Rehabilitation Sciences, The Ohio State University College of Medicine; Emily Hill, MS, LD, RDN, Medical Dietetics \& Health Sciences, School of Health and Rehabilitation Sciences, The Ohio State University College of Medicine; Menglin $X u, P h D$, The Ohio State University Wexner Medical Center; Dennis Cleary, MS, OTD, OTR/L, Division of Occupational Therapy, School of Health and Rehabilitation Sciences, The Ohio State University; Colleen Spees, PhD, LD, RDN, FAND, MEd, spees.11@osu.edu, Medical Dietetics \& Health Sciences, School of Health and Rehabilitation Sciences, The Ohio State University College of Medicine; Comprehensive Cancer Center, Arthur G. James Cancer Hospital and Richard J. Solove Research Institute, $453 \mathrm{~W}$ 10th Ave, Columbus, OH, 43210

Background: Fruit and vegetable consumption is an evidence-based modifiable behavior known to improve physical and mental health for cancer survivors. Understanding sensory perceptions of various produce sources may provide insight into eating behaviors and inform interventions aimed at improving dietary patterns.

Objective: The objective of this study was to assess the sensory perceptions of cancer survivors and their caregivers when asked to evaluate garden-harvested vs grocery-purchased produce.

Study Design, Setting, Participants: Cancer survivors enrolled in a biobehavioral intervention and their caregivers $(n=32)$ participated in a sensory evaluation of 4 produce types sourced from a local garden and a grocery store: tangerine cherry tomatoes, green cabbage, green beans, and green bell peppers. For each type, participants were presented with blinded samples from each source and asked to conduct sensory evaluations.

Measurable Outcome/Analysis: Sensory evaluations included initial preference (garden vs grocery) and liking/ acceptability (9-point hedonic scale) of blinded samples, followed by discrimination between garden- and grocerysource samples (tetrad test) and self-labeling of perceived source and repeat preference and liking/acceptability evaluations. Binomial statistics and Wilcoxon signed rank tests were used to assess differences in evaluations by source for each produce type.

Results: Upon initial blinded evaluation, a significant preference for grocery-purchased produce was noted for green cabbage, green beans, and green bell peppers, but not for tomatoes (all $P<0.05$ ). After self-labeling, participants reported a preference for perceived garden-harvested produce for all produce types (all $P \leq 0.001$ ) even when incorrectly labeled. Liking/acceptability scores were significantly higher among self-labeled garden-harvested produce for all types (all $P \leq 0.001$ ).

Conclusion: These data reveal adult cancer survivors and their caregivers perceive garden-harvested produce as superior in taste, smell, and texture to grocery-purchased even when incorrectly identified. These findings indicate future interventions should address these perceptions to facilitate improvements in produce consumption.

Funding: None.

\section{P34 The Correlation Between Internal Bodily Signals, Eating Behaviors, and Self-Regulation of Food Intake in College Students}

Padideh Lovan,PhD, phadd001@fiu.edu, Florida International University, 11200 SW 8th St Building AHC5, Department of Dietetics and Nutrition, Miami, FL, 33199; Catherine Coccia, $P h D, R D N$, Florida International University

Background: College years are known to be pivotal in adapting to unhealthy eating behaviors. The lack of the ability to recognize physiological needs and insufficient self-regulation in food intake have been reported to be significant determinants in college weight gain. However, the relationship between self-regulation, understanding internal bodily signals, and students eating behaviors are not clear.

Objective: To examine the correlation between self-regulation of food intake, interoception, and eating behaviors in college students living in South Florida.

Study Design, Setting, Participants: Participants were 60 undergraduate college students $($ Mage $=19.8$, SDage $=1.43,37.9 \%$ males, $62.1 \%$ females, $66.6 \%$ Underweight/Normal weight, $33.4 \%$ Overweight/Obese). Interoception and eating behavior data were gathered using questionnaires. Self-regulation was measure using Compensation Indices (COMPX). Students had 2 visits where they were offered a preload drink (either low or high calorie) followed by an ad-libitum buffet lunch to measure the effect of the preloads on students' food intake. Intake was estimated using pictures verified by plate weights.

Measurable Outcome/Analysis: Multiple linear regression analysis was performed to determine the correlation between interoception, eating behaviors, and COMPX. 


\section{P34 (continued)}

Results: When controlled for gender, there were significant correlations between interoception and COMPX $\left(\mathrm{F}=5.56, P=0.002, \mathrm{R}^{2}=0.247\right)$, emotional eating $(\mathrm{F}=$ $\left.3.89, P=0.013, \mathrm{R}^{2}=0.158\right)$, and uncontrolled eating $(\mathrm{F}$ $\left.=3.95, P=0.012, \mathrm{R}^{2}=0.163\right)$. Additionally, COMPX was associated with cognitive restraint eating $(F=8.67$, $\left.P=0.001, \mathrm{R}^{2}=0.250\right)$. When controlled for Body Mass Index (BMI), interoception only had a significant correlation with emotional eating $\left(\mathrm{F}=3.10, P=0.033, \mathrm{R}^{2}=\right.$ $0.131)$, uncontrolled eating $\left(\mathrm{F}=3.06, P=0.035, \mathrm{R}^{2}=\right.$ $0.131)$, and restraint eating $\left(\mathrm{F}=5.35, P=0.002, \mathrm{R}^{2}=\right.$ $0.206)$. There was no correlation between COMPX, eating behaviors, and interoception $(P>0.05)$. No significant mediation effect was observed.

Conclusion: College students who showed higher levels of awareness of internal bodily signals seem to have better self-regulation of food intake. Moreover, self-regulation appears to be negatively affected by the students' emotions and restraint where students show better regulation in food intake when there is less emotional influence and eating restrictions.

Funding: None.

\section{P35 The Effect of a Traffic Light Labeling Intervention on Amount of Food Served in a College Dining Hall}

Amy Martin, Bradley University; Rachel L. Vollmer, PhD, RDN, rvollmer@fsmail.bradley.edu, Bradley University, 1501 W. Bradley Ave, Peoria, IL, 61625

Background: College students may not use the nutrition facts label suggesting changes to the label, such as the Traffic Light Label, are needed to increase user friendliness.

Objective: To examine the effect of traffic light labels on the amount of food served in a university dining hall in comparison to the control nutrition facts panels.

Study Design, Setting, Participants: This study utilized a repeated measures observational design with a control and an intervention period each lasting 28 days at a Midwestern midsize, private university. Following the control period (nutrition facts panels), each food was labeled with a single color (red, yellow, or green) based upon its nutritional quality.

Measurable Outcome/Analysis: Number of servings per day by color (dependent variable) was combined for both lunch and dinner during the control and intervention period. To compare the amount served per day of each color during control and intervention, a one-way analysis of variance (ANOVA) was used. Bonferroni post hoc tests were utilized for multiple comparisons.

Results: The one-way ANOVA for color and time point was significant $(\mathrm{F}(5,150)=4.75, P<0.001)$. Yellowlabeled foods during the intervention $(\mathrm{M}=341.89$, $\mathrm{SD}=275.86)$ was significantly lower than red-labeled foods during control $(\mathrm{M}=654.56, \mathrm{SD}=286.40, P<0.0001)$ and intervention $(\mathrm{M}=604.91, \mathrm{SD}=295.84, P=0.008)$. However, there were no other significant differences between colors and time points.

Conclusion: These results suggest that traffic light labels may not be more effective than nutrition facts panels in college dining halls to improve food choices. Specifically, there was no significant difference in number of servings per day in red- and/or green-labeled foods between control and intervention. Furthermore, servings per day of redlabeled foods continued to be significantly greater than yellow-labeled foods during the intervention. Because students may not use nutrition labels to make food choices, college dining halls should consider reformulating recipes to improve healthfulness of options.

Funding: Bradley University C.C. Wheeler Institute.

\section{P36 The Impact of COVID-19 on Food Security Status and Food Access Among SNAP-Eligible Utahns}

McKenna Voorhees, RDN, Utah State University; Mateja Savoie-Roskos,PhD, MPH, RDN, mateja.savoie@usu.edu, Utah State University, 8700 Old Main Hill, Logan, UT, 84322; Casey Coombs, MS, RDN, Utah State University Extension; Heidi LeBlanc, MS, Utah State University Extension; Heidi Wengreen, PhD, RDN, Utah State University

Background: COVID-19 has the potential to impact the health of individuals experiencing food insecurity and reduced food access. A national collaboration examined this impact across the United States, including Utah.

Objective: The objective of this study was to explore the effect of COVID-19 on perceived food access challenges, barriers, and compensatory strategies among SNAP-eligible Utahns.

Study Design: A 76-item survey was emailed to all SNAPeligible Utahns $(\mathrm{N}=24,763)$ in July 2020. The USDA's 6item Food Security Module was used to determine food security prior to and since COVID-19. Additional questions asked about food access and eating/purchasing behaviors. Participants $(\mathrm{n}=521)$ were predominately White (75\%) females (77\%).

Measurable Outcome/Analysis: Level of agreement for using compensatory food access strategies was summed (score range: 7-42; higher scores reflecting increased use) and assessed for mean differences using ANOVA based on food insecurity classification. Rating scales were used to measure food access challenges and barriers to using nutrition assistance (4-5 item scales) during COVID-19. Spearman correlations examined associations between challenges/barriers and degree of food insecurity. Results: Of participants $(\mathrm{n}=358)$ who were food insecure, 74\% were White and 77\% were female. Food insecure individuals prior to and since COVID-19 were more likely than food secure participants to use compensatory strategies to ensure food affordability during COVID-19 (mean score $=30$ vs 26, respectively; $P=0.002$ ). Degree of 